\title{
Mazur-Ulam theorem under weaker conditions in the framework of 2-fuzzy 2-normed linear spaces
}

\author{
Choonkil Park' and Cihangir Alaca ${ }^{2 *}$
}

\section{"Correspondence:}

cihangiralaca@yahoo.com.tr

${ }^{2}$ Department of Mathematics,

Faculty of Science and Arts, Celal

Bayar University, Manisa, 45140,

Turkey

Full list of author information is

available at the end of the article

\begin{abstract}
The purpose of this paper is to prove that every 2-isometry without any other conditions from a fuzzy 2-normed linear space to another fuzzy 2-normed linear space is affine, and to give a new result of the Mazur-Ulam theorem for 2-isometry in the framework of 2-fuzzy 2-normed linear spaces.
\end{abstract}

MSC: 03E72; 46B20; 51M25; 46B04; 46540

Keywords: $\alpha$-2-norm; 2-fuzzy 2-normed linear spaces; 2-isometry; Mazur-Ulam theorem

\section{Introduction}

A satisfactory theory of 2-norm and $n$-norm on a linear space has been introduced and developed by Gähler in [1, 2]. Freese and Cho [3] gave some isometry conditions in linear 2-normed spaces. Raja and Vaezpour [4] introduced the notion of 2-normed hyperset in a hypervector and also constructed some special 2-normed hypersets of strong homomorphisms over hypervector spaces. Different authors introduced the definitions of fuzzy norms on a linear space. For reference, one may see [5]. Following Cheng and Mordeson [6], Bag and Samanta [7] introduced the concept of fuzzy norm on a linear space.

Somasundaram and Beaula [8] introduced the concept of 2-fuzzy 2-normed linear space or fuzzy 2-normed linear space of the set of all fuzzy sets of a set. They gave the notion of $\alpha$-2-norm on a linear space corresponding to a 2-fuzzy 2-norm with the help of [7] and also gave some fundamental properties of this space.

Let $X$ and $Y$ be metric spaces. A mapping $f: X \rightarrow Y$ is called an isometry if $f$ satisfies $d_{Y}(f(x), f(y))=d_{X}(x, y)$ for all $x, y \in X$, where $d_{X}(\cdot, \cdot)$ and $d_{Y}(\cdot, \cdot)$ denote the metrics in the spaces $X$ and $Y$, respectively. Two metric spaces $X$ and $Y$ are defined to be isometric if there exists an isometry of $X$ onto $Y$. In 1932, Mazur and Ulam [9] proved the following theorem.

Mazur-Ulam theorem Every isometry of a real normed linear space onto a real normed linear space is a linear mapping up to translation.

Baker [10] showed that an isometry from a real normed linear space into a strictly convex real normed linear space is affine. Also, Jian [11] investigated the generalizations of the Mazur-Ulam theorem in $F^{*}$-spaces. Th.M. Rassias and Wagner [12] described all volume

\section{Springer}

(c) 2013 Park and Alaca; licensee Springer. This is an Open Access article distributed under the terms of the Creative Commons Attribution License (http://creativecommons.org/licenses/by/2.0), which permits unrestricted use, distribution, and reproduction in any medium, provided the original work is properly cited. 
preserving mappings from a real finite dimensional vector space into itself and Väisälä [13] gave a short and simple proof of the Mazur-Ulam theorem. Chu [14] proved that the Mazur-Ulam theorem holds when $X$ is a linear 2-normed space. Chu et al. [15] generalized the Mazur-Ulam theorem when $X$ is a linear $n$-normed space, that is, the Mazur-Ulam theorem holds, when the $n$-isometry mapped to a linear $n$-normed space is affine. They also obtained extensions of Th.M. Rassias and Šemrl's theorem [16]. The Mazur-Ulam theorem has been extensively studied by many authors in different aspects (see [12, 1720]).

Recently, Cho et al. [21] investigated the Mazur-Ulam theorem on probabilistic 2normed spaces. Moslehian and Sadeghi [22] investigated the Mazur-Ulam theorem in non-Archimedean spaces. Choy and $\mathrm{Ku}$ [23] proved that the barycenter of a triangle carries the barycenter of a corresponding triangle. They showed the Mazur-Ulam problem on non-Archimedean 2-normed spaces using the above statement. Chen and Song [24] introduced the concept of weak $n$-isometry, and then they got that under some conditions a weak $n$-isometry is also an $n$-isometry. Alaca [25] gave the concepts of 2 -isometry, collinearity, 2-Lipschitz mapping in 2-fuzzy 2-normed linear spaces. Also, he gave a new generalization of the Mazur-Ulam theorem when $X$ is a 2-fuzzy 2-normed linear space or $\Im(X)$ is a fuzzy 2-normed linear space. Park and Alaca [26] introduced the concept of 2 -fuzzy $n$-normed linear space or fuzzy $n$-normed linear space of the set of all fuzzy sets of a non-empty set. They defined the concepts of $n$-isometry, $n$-collinearity, $n$-Lipschitz mapping in this space. Also, they generalized the Mazur-Ulam theorem, that is, when $X$ is a 2 -fuzzy $n$-normed linear space or $\Im(X)$ is a fuzzy $n$-normed linear space, the MazurUlam theorem holds. Moreover, it is shown that each $n$-isometry in 2 -fuzzy $n$-normed linear spaces is affine. Ren [27] showed that every generalized area $n$ preserving mapping between real 2-normed linear spaces $X$ and $Y$ which is strictly convex is affine under some conditions.

In the present paper, we give a new version of Mazur-Ulam theorem with a new method when $X$ is a 2-fuzzy 2-normed linear space or $\Im(X)$ is a fuzzy 2-normed linear space.

\section{On 2-fuzzy 2-normed linear spaces}

In this section, at first we give the concept of linear 2-normed space and later the concept of 2-fuzzy 2-normed linear space and its fundamental properties with help of [8]. For more details, we refer the readers to $[7,8,28,29]$.

Definition 2.1 [28] Let $X$ be a real vector space of dimension greater than 1 and let $\|\bullet, \bullet\|$ be a real-valued function on $X \times X$ satisfying the following four properties:

(1) $\|x, y\|=0$ if and only if $x$ and $y$ are linearly dependent,

(2) $\|x, y\|=\|y, x\|$,

(3) $\|x, \alpha y\|=|\alpha|\|x, y\|$ for any $\alpha \in \mathbb{R}$,

(4) $\|x, y+z\| \leq\|x, y\|+\|x, z\|$,

$\|\bullet, \bullet\|$ is called a 2-norm on $X$ and the pair $(X,\|\bullet, \bullet\|)$ is called a linear 2-normed space.

Definition 2.2 [7] Let $X$ be a linear space over $S$ (a field of real or complex numbers). A fuzzy subset $N$ of $X \times \mathbb{R}$ (R, the set of real numbers) is called a fuzzy norm on $X$ if and only if:

(N1) For all $t \in \mathbb{R}$ with $t \leq 0, N(x, t)=0$, 
(N2) For all $t \in \mathbb{R}$ with $t>0, N(x, t)=1$ if and only if $x=0$,

(N3) For all $t \in \mathbb{R}$ with $t>0, N(\lambda x, t)=N\left(x, \frac{t}{|\lambda|}\right)$, if $\lambda \neq 0, \lambda \in S$,

(N4) For all $s, t \in \mathbb{R}, x, y \in X, N(x+y, s+t) \geq \min \{N(x, s), N(y, t)\}$,

(N5) $N(x, \cdot)$ is a non-decreasing function of $t \in \mathbb{R}$ and $\lim _{t \rightarrow \infty} N(x, t)=1$.

Then $(X, N)$ is called a fuzzy normed linear space or, in short, $\mathrm{f}$-NLS.

Theorem 2.1 [7] Let $(X, N)$ be an $f$-NLS. Assume the condition that

(N6) $N(x, t)>0$ for all $t>0$ implies $x=0$.

Define $\|x\|_{\alpha}=\inf \{t: N(x, t) \geq \alpha\}, \alpha \in(0,1)$. Then $\left\{\|\bullet\|_{\alpha}: \alpha \in(0,1)\right\}$ is an ascending family of norms on $X$. We call these norms $\alpha$-norms on $X$ corresponding to the fuzzy norm on $X$.

Definition 2.3 Let $X$ be any non-empty set and $\Im(X)$ be the set of all fuzzy sets on $X$. For $U, V \in \Im(X)$ and $\lambda \in S$ the field of real numbers, define

$$
U+V=\{(x+y, \nu \wedge \mu):(x, v) \in U,(y, \mu) \in V\}
$$

and $\lambda U=\{(\lambda x, v):(x, v) \in U\}$.

Definition 2.4 A fuzzy linear space $\widehat{X}=X \times(0,1]$ over the number field $S$, where the addition and scalar multiplication operation on $X$ are defined by $(x, v)+(y, \mu)=(x+y, \nu \wedge$ $\mu), \lambda(x, v)=(\lambda x, v)$ is a fuzzy normed space if to every $(x, v) \in \widehat{X}$, there is associated a nonnegative real number, $\|(x, v)\|$, called the fuzzy norm of $(x, v)$, in such a way that

(i) $\|(x, v)\|=0$ iff $x=0$ the zero element of $X, v \in(0,1]$,

(ii) $\|\lambda(x, v)\|=|\lambda|\|(x, v)\|$ for all $(x, v) \in \widehat{X}$ and all $\lambda \in S$,

(iii) $\|(x, v)+(y, \mu)\| \leq\|(x, v \wedge \mu)\|+\|(y, v \wedge \mu)\|$ for all $(x, v),(y, \mu) \in \widehat{X}$,

(iv) $\left\|\left(x, \bigvee_{t} v_{t}\right)\right\|=\bigwedge_{t}\left\|\left(x, v_{t}\right)\right\|$ for all $v_{t} \in(0,1]$

Definition 2.5 [8] Let $X$ be a non-empty and $\Im(X)$ be the set of all fuzzy sets in $X$. If $f \in$ $\Im(X)$, then $f=\{(x, \mu): x \in X$ and $\mu \in(0,1]\}$. Clearly, $f$ is a bounded function for $|f(x)| \leq 1$. Let $S$ be the space of real numbers, then $\Im(X)$ is a linear space over the field $S$ where the addition and multiplication are defined by

$$
f+g=\{(x, \mu)+(y, \eta)\}=\{(x+y, \mu \wedge \eta):(x, \mu) \in f \text { and }(y, \eta) \in g\}
$$

and

$$
\lambda f=\{(\lambda x, \mu):(x, \mu) \in f\},
$$

where $\lambda \in S$.

The linear space $\Im(X)$ is said to be a normed space if for every $f \in \Im(X)$, there is associated a non-negative real number $\|f\|$ called the norm of $f$ in such a way that

(i) $\|f\|=0$ if and only if $f=0$. For

$$
\begin{aligned}
\|f\| & =0 \\
& \Longleftrightarrow \quad\{\|(x, \mu)\|:(x, \mu) \in f\}=0 \\
& \Longleftrightarrow \quad x=0, \quad \mu \in(0,1] \quad \Longleftrightarrow f=0 .
\end{aligned}
$$


(ii) $\|\lambda f\|=|\lambda|\|f\|, \lambda \in S$. For

$$
\begin{aligned}
\|\lambda f\| & =\{\|\lambda(x, \mu)\|:(x, \mu) \in f, \lambda \in S\} \\
& =\{|\lambda|\|(x, \mu)\|:(x, \mu) \in f\}=|\lambda|\|f\| .
\end{aligned}
$$

(iii) $\|f+g\| \leq\|f\|+\|g\|$ for every $f, g \in \Im(X)$. For

$$
\begin{aligned}
\|f+g\| & =\{\|(x, \mu)+(y, \eta)\|: x, y \in X, \mu, \eta \in(0,1]\} \\
& =\{\|(x+y),(\mu \wedge \eta)\|: x, y \in X, \mu, \eta \in(0,1]\} \\
& =\{\|(x, \mu \wedge \eta)\|+\|(y, \mu \wedge \eta)\|:(x, \mu) \in f,(y, \eta) \in g\} \\
& =\|f\|+\|g\| .
\end{aligned}
$$

Then $(\Im(X),\|\bullet\|)$ is a normed linear space.

Definition 2.6 [8] A 2-fuzzy set on $X$ is a fuzzy set on $\Im(X)$.

Definition 2.7 [8] Let $\Im(X)$ be a linear space over the real field $S$. A fuzzy subset $N$ of $\Im(X) \times \Im(X) \times \mathbb{R}(\mathbb{R}$, a set of real numbers) is called a 2-fuzzy 2-norm on $X$ (or a fuzzy 2 -norm on $\Im(X))$ if and only if

(2-N1) for all $t \in \mathbb{R}$ with $t \leq 0, N\left(f_{1}, f_{2}, t\right)=0$,

(2-N2) for all $t \in \mathbb{R}$ with $t>0, N\left(f_{1}, f_{2}, t\right)=1$ if and only if $f_{1}$ and $f_{2}$ are linearly dependent,

(2-N3) $N\left(f_{1}, f_{2}, t\right)$ is invariant under any permutation of $f_{1}, f_{2}$,

(2-N4) for all $t \in \mathbb{R}$ with $t>0, N\left(f_{1}, \lambda f_{2}, t\right)=N\left(f_{1}, f_{2}, \frac{t}{|\lambda|}\right)$, if $\lambda \neq 0, \lambda \in S$,

(2-N5) for all $s, t \in \mathbb{R}$,

$$
N\left(f_{1}, f_{2}+f_{3}, s+t\right) \geq \min \left\{N\left(f_{1}, f_{2}, s\right), N\left(f_{1}, f_{3}, t\right)\right\},
$$

$(2-\mathrm{N} 6) N\left(f_{1}, f_{2}, \cdot\right):(0, \infty) \rightarrow[0,1]$ is continuous,

(2-N7) $\lim _{t \rightarrow \infty} N\left(f_{1}, f_{2}, t\right)=1$.

Then $(\Im(X), N)$ is a fuzzy 2-normed linear space or $(X, N)$ is a 2-fuzzy 2-normed linear space.

Remark 2.1 In a 2-fuzzy 2-normed linear space $(X, N), N\left(f_{1}, f_{2}, \cdot\right)$ is a non-decreasing function of $\mathbb{R}$ for all $f_{1}, f_{2} \in \Im(X)$.

Theorem 2.2 [8] Let $(\Im(X), N)$ be a fuzzy 2-normed linear space. Assume that (2-N8) $N\left(f_{1}, f_{2}, t\right)>0$ for all $t>0$ implies $f_{1}$ and $f_{2}$ are linearly dependent.

Define $\left\|f_{1}, f_{2}\right\|_{\alpha}=\inf \left\{t: N\left(f_{1}, f_{2} t\right) \geq \alpha, \alpha \in(0,1)\right\}$.

Then $\left\{\|\bullet, \bullet\|_{\alpha}: \alpha \in(0,1)\right\}$ is an ascending family of 2-norms on $\Im(X)$. These 2-norms are called $\alpha$-2-norms on $\Im(X)$ corresponding to the 2-fuzzy 2-norm on $X$.

\section{On the Mazur-Ulam theorem}

Recently, Alaca [25] introduced the concept of 2-isometry which is suitable to represent the notion of area-preserving mappings in fuzzy 2 -normed linear spaces as follows. 
For $f, g, h \in \Im(X)$ and $\alpha, \beta \in(0,1),\|f-h, g-h\|_{\alpha}$ is called an area of $f, g$ and $h$. We call $\Psi$ a 2-isometry if $\|f-h, g-h\|_{\alpha}=\|\Psi(f)-\Psi(h), \Psi(g)-\Psi(h)\|_{\beta}$ for all $f, g, h \in \Im(X)$ and $\alpha, \beta \in(0,1)$.

A version of the Mazur-Ulam theorem has been obtained in [25] as follows.

Theorem 3.1 [25] Assume that $\Im(X)$ and $\Im(Y)$ are fuzzy 2-normed linear spaces. If $\Psi$ : $\Im(X) \rightarrow \Im(Y)$ is a 2-isometry and satisfies $\Psi(f), \Psi(g)$ and $\Psi(h)$ are collinear when $f, g$ and $h$ are collinear, then $\Psi$ is affine.

A natural question is whether the 2-isometry in the fuzzy 2-normed linear spaces is also affine without the condition of preserving collinearity. In this section, we find a reply to this question when $X$ is a 2-fuzzy 2-normed linear space or $\Im(X)$ is a fuzzy 2 -normed linear space.

Lemma $3.1[25]$ For all $f, g \in \Im(X), \alpha \in(0,1)$ and $\lambda \in \mathbb{R}$. Then

$$
\|f, g\|_{\alpha}=\|f, g+\lambda f\|_{\alpha} .
$$

Lemma 3.2 Let $f, g, h \in \Im(X)$ and $\alpha \in(0,1)$. Then $v=\frac{f+g}{2}$ is the unique element of $\Im(X)$ satisfying

$$
\|f-h, f-v\|_{\alpha}=\|g-v, g-h\|_{\alpha}=\frac{1}{2}\|f-h, g-h\|_{\alpha}
$$

with $\|f-h, g-h\|_{\alpha} \neq 0$ and $v \in\{k f+(1-k) g: k \in \mathbb{R}\}$.

Proof From Lemma 3.1, it is obvious that $v=\frac{f+g}{2}$ satisfies

$$
\|f-h, f-v\|_{\alpha}=\|g-v, g-h\|_{\alpha}=\frac{1}{2}\|f-h, g-h\|_{\alpha}
$$

with $\|f-h, g-h\|_{\alpha} \neq 0$ and $v \in\{k f+(1-k) g: k \in \mathbb{R}\}$.

For the uniqueness of $v$, assume that $u \in \Im(X)$ also satisfies

$$
\|f-h, f-u\|_{\alpha}=\|g-u, g-h\|_{\alpha}=\frac{1}{2}\|f-h, g-h\|_{\alpha}
$$

with $\|f-h, g-h\|_{\alpha} \neq 0$ and $u \in\{k f+(1-k) g: k \in \mathbb{R}\}$. Let $u=k f+(1-k) g$ for some $k \in \mathbb{R}$. From Lemma 3.1, we have

$$
\begin{aligned}
\|f-h, g-h\|_{\alpha} & =2\|f-h, f-u\|_{\alpha} \\
& =2\|f-h, f-(k f+(1-k) g)\|_{\alpha} \\
& =2|1-k|\|f-h, f-g\|_{\alpha} \\
& =2|1-k|\|f-h, g-h\|_{\alpha}
\end{aligned}
$$

and

$$
\begin{aligned}
\|f-h, g-h\|_{\alpha} & =2\|g-h, g-u\|_{\alpha} \\
& =2\|g-h, g-(k f+(1-k) g)\|_{\alpha}
\end{aligned}
$$




$$
\begin{aligned}
& =2|k|\|g-h, g-f\|_{\alpha} \\
& =2|k|\|f-h, g-h\|_{\alpha}
\end{aligned}
$$

Since $\|f-h, g-h\|_{\alpha} \neq 0$, we have $1=2|1-k|=2|k|$. So, $k=\frac{1}{2}$ and $u=v=\frac{f+g}{2}$.

Theorem 3.2 Let $\Im(X)$ and $\Im(Y)$ be fuzzy 2-normed linear spaces. If $\Psi: \Im(X) \rightarrow \Im(Y)$ is a 2-isometry, then $\Psi$ is affine.

Proof Let $\Phi(f)=\Psi(f)-\Psi(0)$. Obviously, $\Phi(0)=0$ and $\Phi$ is a 2-isometry. Now, we prove that $\Phi$ is linear.

Firstly, we show that $\Phi$ is additive. For $f, g, h \in \Im(X), \alpha, \beta \in(0,1)$ with $\|f-h, g-h\|_{\alpha} \neq 0$, $\|\Phi(f)-\Phi(h), \Phi(g)-\Phi(h)\|_{\beta} \neq 0$ and from Lemma 3.1, we have

$$
\begin{aligned}
\left\|\Phi(f)-\Phi(h), \Phi(f)-\Phi\left(\frac{f+g}{2}\right)\right\|_{\beta} & =\left\|f-h, f-\frac{f+g}{2}\right\|_{\alpha} \\
& =\left\|f-h, \frac{f-g}{2}\right\|_{\alpha} \\
& =\frac{1}{2}\|f-h, f-g\|_{\alpha} \\
& =\frac{1}{2}\|f-h, g-h\|_{\alpha} \\
& =\frac{1}{2}\|\Phi(f)-\Phi(h), \Phi(g)-\Phi(h)\|_{\beta} .
\end{aligned}
$$

Similarly,

$$
\left\|\Phi(g)-\Phi(h), \Phi(g)-\Phi\left(\frac{f+g}{2}\right)\right\|_{\beta}=\frac{1}{2}\|\Phi(f)-\Phi(h), \Phi(g)-\Phi(h)\|_{\beta} .
$$

And

$$
\begin{aligned}
\left\|\Phi\left(\frac{f+g}{2}\right)-\Phi(g), \Phi(f)-\Phi(g)\right\|_{\beta} & =\left\|\frac{f+g}{2}-g, f-g\right\|_{\alpha} \\
& =\frac{1}{2}\|f-g, f-g\|_{\alpha}=0 .
\end{aligned}
$$

So, we get

$$
\Phi\left(\frac{f+g}{2}\right)-\Phi(g)=k(\Phi(f)-\Phi(g))
$$

for some $k \in \mathbb{R}$ by Definition 2.7. That is,

$$
\Phi\left(\frac{f+g}{2}\right)=k \Phi(f)+(1-k) \Phi(g)
$$

Thus, from Lemma 3.2,

$$
\Phi\left(\frac{f+g}{2}\right)=\frac{\Phi(f)+\Phi(g)}{2}
$$

for all $f, g \in \Im(X)$. 
Since $\Phi(0)=0$, we have

$$
\Phi\left(\frac{f}{2}\right)=\Phi\left(\frac{f+0}{2}\right)=\frac{\Phi(f)+\Phi(0)}{2}=\frac{\Phi(f)}{2}
$$

and

$$
\begin{aligned}
\Phi(f+g) & =\Phi\left(\frac{2 f+2 g}{2}\right)=\frac{\Phi(2 f)+\Phi(2 g)}{2}=\frac{\Phi(2 f)}{2}+\frac{\Phi(2 g)}{2} \\
& =\Phi(f)+\Phi(g) .
\end{aligned}
$$

It follows that $\Phi$ is additive.

Secondly, we show that $\Phi(r f)=r \Phi(f)$ for every $r \in \mathbb{R}, f \in \Im(X)$ and $\alpha, \beta \in(0,1)$. Let $r \in \mathbb{R}^{+}$and $f \in \Im(X)$ and $\alpha, \beta \in(0,1)$. Since $\Phi(0)=0$ and $\Phi$ is a 2 -isometry, we have

$$
\begin{aligned}
\|\Phi(r f), \Phi(f)\|_{\beta} & =\|\Phi(r f)-\Phi(0), \Phi(f)-\Phi(0)\|_{\beta} \\
& =\|r f-0, f-0\|_{\alpha} \\
& =\|r f, f\|_{\alpha} \\
& =0 .
\end{aligned}
$$

So, $\Phi(r f)=s \Phi(f)$ for some $s \in \mathbb{R}$ from Definition 2.7. As $\operatorname{dim} \Im(X)>1$, there exists a $g \in$ $\Im(X)$ such that $\|f, g\|_{\alpha} \neq 0$. It is easy to see that

$$
\begin{aligned}
r\|f, g\|_{\alpha} & =\|r f, g\|_{\alpha}=\|\Phi(r f), \Phi(g)\|_{\beta}=\|s \Phi(f), \Phi(g)\|_{\beta} \\
& =|s|\|\Phi(f), \Phi(g)\|_{\beta}=|s|\|f, g\|_{\alpha} .
\end{aligned}
$$

So, $s=r$ or $s=-r$. If $s=-r$, then

$$
\begin{aligned}
|r-1|\|f, g\|_{\alpha} & =\|(r-1) f, g\|_{\alpha}=\|r f-f, g-0\|_{\alpha} \\
& =\|\Phi(r f)-\Phi(f), \Phi(g)-\Phi(0)\|_{\beta} \\
& =\|-r \Phi(f)-\Phi(f), \Phi(g)\|_{\beta} \\
& =(r+1)\|\Phi(f), \Phi(g)\|_{\beta} \\
& =(r+1)\|f, g\|_{\alpha} .
\end{aligned}
$$

So, $|r-1|=r+1$. This is a contradiction since $r \in \mathbb{R}^{+}$. Thus, $\Phi(r f)=r \Phi(f)$ for every $r \in \mathbb{R}^{+}$, $f \in \Im(X)$ and $\alpha, \beta \in(0,1)$.

Similarly, we can prove $\Phi(r f)=r \Phi(f)$ for every $r \in \mathbb{R}^{-}, f \in \Im(X)$ and $\alpha, \beta \in(0,1)$.

Hence, we prove that $\Phi$ is linear and $\Psi$ is affine.

Remark 3.1 Theorem 3.1 has been substantially improved by Theorem 3.2.

Remark 3.2 It is clear that the Mazur-Ulam theorem has been proved under much weaker conditions than the main result of Alaca [25] in the framework of 2-fuzzy 2-normed linear spaces. 
Open problem How can obtain some results for the Aleksandrov problem in fuzzy 2normed linear spaces with the help of this technique?

\section{Competing interests}

The authors declare that they have no competing interests.

\section{Authors' contributions}

All authors conceived of the study, participated in its design and coordination, drafted the manuscript, participated in the sequence alignment, and read and approved the final manuscript.

\section{Author details}

'Department of Mathematics, Research Institute for Natural Sciences, Hanyang University, Seoul, 133-791, South Korea.

2Department of Mathematics, Faculty of Science and Arts, Celal Bayar University, Manisa, 45140, Turkey.

\section{Acknowledgements}

The authors would like to thank the referees and the area editor Professor Yeol Je Cho for their valuable suggestions and comments.

\section{Received: 10 September 2012 Accepted: 6 January 2013 Published: 28 February 2013}

\section{References}

1. Gähler, S: Lineare 2-normierte raume. Math. Nachr. 28, 1-43 (1964). doi:10.1002/mana.19640280102

2. Gähler, S: Untersuchungen über verallgemeinerte m-metrische räume I. Math. Nachr. 40, 165-189 (1969). doi:10.1002/mana.19690400114

3. Freese, RW, Cho, YJ: Isometry conditions in linear 2-normed spaces. Math. Jpn. 35(5), 1-6 (1990)

4. Raja, P, Vaezpour, SM: On 2-strong homomorphisms and 2-normed hypersets in hypervector spaces. J. Nonlinear Sci. Appl. 1(4), 213-223 (2008)

5. Felbin, C: Finite-dimensional fuzzy normed linear space. Fuzzy Sets Syst. 48(2), 239-248 (1992)

6. Cheng, SC, Mordeson, JN: Fuzzy linear operators and fuzzy normed linear spaces. Bull. Calcutta Math. Soc. 86, 429-436 (1994)

7. Bag, T, Samanta, SK: Finite dimensional fuzzy normed linear spaces. J. Fuzzy Math. 11, 687-705 (2003)

8. Somasundaram, RM, Beaula, T: Some aspects of 2-fuzzy 2-normed linear spaces. Bull. Malays. Math. Soc. 32, 211-221 (2009)

9. Mazur, S, Ulam, S: Sur les transformationes isomé triques d'espaces vectoriels normés. C. R. Math. Acad. Sci. Paris 194, 946-948 (1932)

10. Baker, JA: Isometries in normed spaces. Am. Math. Mon. 78, 655-658 (1971). doi:10.2307/2316577

11. Jian, W: On the generations of the Mazur-Ulam isometric theorem. J. Math. Anal. Appl. 263, 510-521 (2001). doi:10.1006/jmaa.2001.7627

12. Rassias, TM, Wagner, P: Volume preserving mappings in the spirit of the Mazur-Ulam theorem. Aequ. Math. 66, 85-89 (2003). doi:10.1007/s00010-003-2669-7

13. Väisälä, J: A proof of the Mazur-Ulam theorem. Am. Math. Mon. 110, 633-635 (2003). doi:10.2307/3647749

14. Chu, H: On the Mazur-Ulam problem in linear 2-normed spaces. J. Math. Anal. Appl. 327, 1041-1045 (2007). doi:10.1016/j.jmaa.2006.04.053

15. Chu, H, Choi, S, Kang, D: Mappings of conservative distances in linear n-normed spaces. Nonlinear Anal. 70, 1168-1174 (2009). doi:10.1016/j.na.2008.02.002

16. Rassias, TM, Šemrl, P: On the Mazur-Ulam problem and the Aleksandrov problem for unit distance preserving mappings. Proc. Am. Math. Soc. 118, 919-925 (1993). doi:10.1090/S0002-9939-1993-1111437-6

17. Elhoucien, E, Youssef, M: On the paper A. Najati and S.-M. Jung: the Hyers-Ulam stability of approximately quadratic mappings on restricted domains. J. Nonlinear Anal. Appl. 2012, Article ID jnaa-00127 (2012). doi:10.5899/2012/jnaa-00127

18. Kim, GH: Stability of the Lobacevski equation. J. Nonlinear Sci. Appl. 4(1), 11-18 (2011)

19. Rassias, TM: On the A.D. Aleksandrov problem of conservative distances and the Mazur-Ulam theorem. Nonlinear Anal. 47, 2597-2608 (2001). doi:10.1016/S0362-546X(01)00381-9

20. Xiang, S: Mappings of conservative distances and the Mazur-Ulam theorem. J. Math. Anal. Appl. 254, $262-274$ (2001). doi:10.1006/jmaa.2000.7276

21. Cho, YJ, Rahbarnia, F, Saadati, R, Sadeghi, Gh: Isometries in probabilistic 2-normed spaces. J. Chungcheong Math. Soc. 22, 623-634 (2009)

22. Moslehian, MS, Sadeghi, Gh: A Mazur-Ulam theorem in non-Archimedean normed spaces. Nonlinear Anal. 69 3405-3408 (2008). doi:10.1016/j.na.2007.09.023

23. Choy, J, Ku, S: Characterization on 2-isometries in non-Archimedean 2-normed spaces. J. Chungcheong Math. Soc. 22, 65-71 (2009)

24. Chen, $X Y$, Song, MM: Characterizations on isometries in linear n-normed spaces. Nonlinear Anal. 72, 1895-1901 (2010). doi:10.1016/j.na.2009.09.029

25. Alaca, C: A new perspective to the Mazur-Ulam problem in 2-fuzzy 2-normed linear spaces. Iranian J. Fuzzy Syst. 7 109-119(2010)

26. Park, C, Alaca, C: An introduction to 2-fuzzy n-normed linear spaces and a new perspective to the Mazur-Ulam problem. J. Inequal. Appl. 2012, 14 (2012). doi:10.1186/1029-242X-2012-14

27. Ren, W: On the generalized 2-isometry. Rev. Mat. Complut. 23, 97-104 (2010)

28. Cho, YJ, Lin, PCS, Kim, SS, Misiak, A: Theory of 2-Inner Product Spaces. Nova Science Publishers, New York (2001)

29. Freese, RW, Cho, YJ: Geometry of Linear 2-Normed Spaces. Nova Science Publishers, New York (2001) 
doi:10.1186/1029-242X-2013-78

Cite this article as: Park and Alaca: Mazur-Ulam theorem under weaker conditions in the framework of 2-fuzzy

2-normed linear spaces. Journal of Inequalities and Applications 2013 2013:78.

Submit your manuscript to a SpringerOpen ${ }^{\circ}$ journal and benefit from:

- Convenient online submission

- Rigorous peer review

- Immediate publication on acceptance

Open access: articles freely available online

- High visibility within the field

- Retaining the copyright to your article

Submit your next manuscript at $\gg$ springeropen.com 